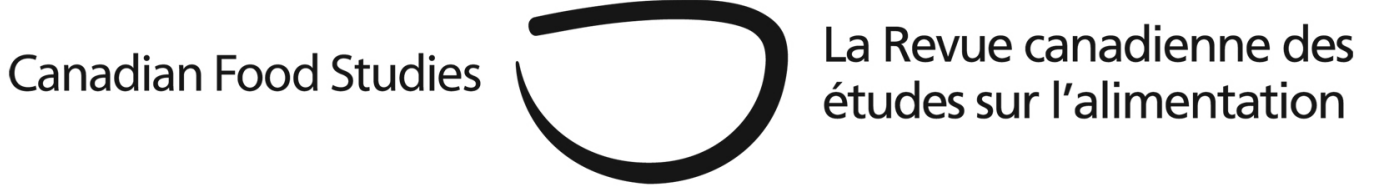

Article de recherche

\title{
Petits commerces de bouche et réseaux alimentaires alternatifs: un regard montréalais
}

\author{
Alexandre Maltais* \\ Chercheur postdoctoral, Faculté de l'aménagement, Université de Montréal
}

\section{Résumé}

Cet article aborde les réseaux de distribution alimentaire alternatifs par l'une de leurs extrémités jusqu’ici négligées dans la littérature comme dans le débat public: les petits commerces de détail urbains. Ceux-ci se multiplient sur plusieurs rues commerçantes dans les grandes villes depuis quelques décennies, à la faveur d'un embourgeoisement de la population des quartiers limitrophes. Cette gentrification entraîne une sophistication des préférences de consommation et favorise un repositionnement des petites entreprises commerciales vers des créneaux plus pointus, à l'abri d'une grande distribution en croissance. Sur la base d'une enquête de terrain de deux ans et d'une trentaine d'entrevues semi-directives réalisées auprès de propriétaires d'établissements commerciaux dont l'offre est entièrement ou partiellement composée de produits ou de services alimentaires, l'article examine les stratégies déployées par les commerçants pour se constituer comme alternatifs, les avantages d'un tel positionnement et les difficultés qui l'accompagnent. Producteurs et distributeurs alternatifs constituent ainsi deux maillons d'une chaîne qui ne peut donc être qualifiée d'alternative que tant qu'elle s'attache à se démarquer de l'univers de la consommation de masse.

Mots-clés: commerce de detail; distribution; alimentation; réseaux alimentaires alternatifs; petit commerce 


\section{Introduction}

Les débats et les travaux sur les réseaux alimentaires dits « alternatifs » ont généralement mis l'accent sur la vente directe et les circuits courts de distribution comme les marchés fermiers ou l'agriculture soutenue par la communauté, faisant souvent l'impasse sur des modes de distribution sans doute moins champêtres ou novateurs, mais tout aussi importants dans le développement de filières agroalimentaires non industrielles et dans l'émergence de nouvelles cultures gastronomiques locales (Lemasson, 2006). Tel est notamment le cas des petits commerces de bouche, dont le nombre augmente à nouveau au Québec après des décennies de déclin (Groupe Marché, 2007). Ce renouveau des boucheries, fromageries, boulangeries et autres épiceries fines s'est ainsi fait en parallèle avec le développement d'une agriculture elle aussi qualifiée d'alternative, dont les petits commerces spécialisés constituent souvent l'un des principaux points d'écoulement - comme le soulignait d'ailleurs le rapport de la Commission sur l'avenir de l'agriculture et de l'agroalimentaire québécois, communément appelé rapport Pronovost (CAAAQ, 2008). Néanmoins, les recherches sur la commercialisation de ces produits ont à ce jour disproportionnellement fait état de la situation des producteurs (Bérard \& Marchenay, 2004; Guthman, 2004; Paxson, 2013; Trubek, 2009) ou des consommateurs (Johnston \& Baumann, 2010), sans que ne soit réellement éclairé et explicité le rôle pourtant fondamental des distributeurs (Blay-Palmer \& Donald, 2006), et notamment des détaillants.

Au Québec, en dépit de travaux fondateurs sur les nouvelles filières agricoles de terroir, notamment vinicole (De Koninck, 1993) ou fromagère (Boulianne, 2010; 2013), le caractère alternatif de ces nouveaux modes de distribution reste à ce jour peu défini et souvent pris pour acquis dans les milieux pratiques. Ainsi, lorsqu'il évoque la « distribution alimentaire alternative », le ministère québécois de l’Agriculture des Pêcheries et de l'Alimentation se contente de nommer cinq structures de commercialisation des produits agricoles: les marchés publics, les kiosques à la ferme et l'autocueillette, l'agrotourisme, le commerce électronique et l'agriculture soutenue par la communauté (Ministère de l'Agriculture, des Pêcheries et de l'Alimentation, 2015). Sans expliciter les critères permettant de qualifier ces structures d'alternatives, on les distingue tout de même clairement, dans l'usage, des petites boutiques spécialisées comme les boucheries (Robitaille, 2012, p. 7). Le ministère précise bien que les consommateurs choisissent ces modes de distribution pour trois raisons: avoir un contact plus direct avec les producteurs; soutenir la production agricole locale et régionale; acquérir des produits frais — insistant par là même sur la grande proximité qu'ils établissent entre les producteurs et les consommateurs. Mais les liens entre ces motivations et les cinq structures évoquées apparaissent pour le moins fragiles. Il est en effet possible d'acquérir des produits frais et régionaux dans la plupart des petits établissements commerciaux et même dans plusieurs supermarchés, en particulier chez les indépendants affiliés, qui comptaient au Québec pour 59,5 pourcent du volume des ventes au détail réalisées dans les commerces alimentaires généralistes en 2015 (Condon, 2016, Mars, p. 28). En outre, s’agissant de marchés publics et non strictement fermiers, le contact avec le producteur n'apparaît pas toujours aussi direct que ne le suggèrent les 
représentations souvent idéalisées de cet équipement ancien (La Pradelle, 1996; Tunbridge, 2001). À Montréal notamment, les revendeurs de fruits et de légumes sont nombreux sur les marchés, au moins depuis le dix-neuvième siècle (Bergeron, 1990; Brouillette, 1991). C'est sans compter les bouchers qui ne sont que très exceptionnellement producteurs de leur viande, les boulangers qui fabriquent souvent leur pain dans l'arrière-boutique ou les restaurateurs qui effectuent une partie significative du travail de production du service « repas au restaurant » et qui donnent au passage une grande visibilité aux produits locaux et artisanaux. L'engouement actuel pour la proximité et le local (Hess, 2009; Savage, Bagnall, \& Longhurst, 2005) semble donc avoir favorisé le maintien de représentations et même de définitions officielles parfois en décalage avec la réalité empirique de ces filières. ${ }^{1}$ Car on voit bien, à la lumière des quelques exemples précités, à quel point la production et la distribution des produits alimentaires alternatifs ne sont en réalité que deux segments d'une même chaîne, tout au long de laquelle tant le caractère alternatif que sa valeur marchande sont créés avant d'atteindre la sphère de la consommation (Callon, Méadel, \& Rabeharisoa, 2000).

Dans ce contexte, il apparaît nécessaire de mieux baliser et de mieux comprendre le caractère alternatif des nouveaux réseaux de distribution alimentaire. C'est l'objectif principal de cet article. Nous le ferons du point de vue d'un acteur jusqu'ici négligé, le petit commerçant urbain. Nous examinerons d'abord les logiques qui amènent plusieurs d'entre eux à se constituer et à se présenter comme alternatifs, c'est-à-dire à se distancier, dans la réalité comme dans les discours, des pratiques couramment utilisées par la grande distribution, en faisant notamment affaire avec des réseaux de petits fournisseurs plutôt qu'avec des grossistes. Il s'agira ensuite d'examiner les difficultés propres à ce type de pratiques. Nous conclurons en abordant la nature des liens entre les petits producteurs et les petits commerçants, de manière à circonscrire le rôle que ces derniers peuvent jouer dans le développement d'une production agricole alternative, à la lumière de leurs trajectoires et de leurs réalités professionnelles respectives.

Nous faisons donc l'hypothèse qu'il existe une homologie de position entre les petits commerçants alternatifs et les petits producteurs alternatifs. Nous verrons en effet que le mouvement de polarisation entre une grande distribution généraliste et des petits établissements de plus en plus spécialisés fait écho à celui survenu au sein de la production agricole entre une industrie agroalimentaire standardisée et un nombre croissant de petits producteurs artisanaux, dont la voix est portée au Québec par des organismes comme l'Union Paysanne et Solidarité rurale. Ils occupent ainsi des positions analogues sur leurs marchés respectifs, des positions que l'on qualifie habituellement de « challengers » en sociologie économique (Fligstein, 1996): leur raison d'être est la remise en question de leurs concurrents qui dominent quantitativement le marché. En outre, ils partagent souvent un même rejet de la production de masse, une volonté commune de s'affranchir de ce que Baudrillard (1972, p. 29) appelait les «stigmates de la production industrielle ». Ils cherchent ainsi, chacun à sa manière, à faire « contrepoids aux

\footnotetext{
${ }^{1}$ Tel est également le cas de l'association des marchés publics du Québec qui considère ses activités de distribution comme « une extension des activités de production et de transformation à la ferme » (Association des marchés publics du Québec, 2014, p. 7)
} 
tendances globalisatrices associées à des phénomènes néfastes sur les plans sociaux et environnementaux » (Boulianne, 2010, para. 15).

\section{Méthodologie}

Cet article s’appuie sur une enquête de terrain de deux ans, réalisée entre l'automne 2011 et l'été 2013 sur deux rues commerçantes du centre de Montréal: la rue Ontario Est, dans l'arrondissement Mercier-Hochelaga-Maisonneuve et la rue Notre-Dame Ouest, dans l'arrondissement Sud-Ouest. Ces deux secteurs traditionnellement ouvriers ont notamment été choisis parce que la part relative du commerce de bouche y a considérablement augmenté dans les dernières décennies. Dans le commerce des biens, les effectifs de la catégorie alimentaire ont ainsi augmenté de 10,3 pourcent sur la rue Notre-Dame et d'un remarquable 40 pourcent sur la rue Ontario entre 1971 et $2011 .^{2}$ Dans le domaine des services, on observe une augmentation encore plus importante de la catégorie restauration pendant la même période: 65,6 pourcent dans le Sud-Ouest, 104,2 pourcent dans Hochelaga-Maisonneuve.

Cette prépondérance du commerce de bouche s’explique d'abord par la présence dans les deux quartiers d’un marché public municipal, un équipement longtemps tombé en désuétude qui jouit aujourd'hui d'un regain de popularité, particulièrement auprès des populations urbaines soucieuses de l'environnement et de leur alimentation (Gonzalez \& Waley, 2013; Johnston \& Baumann, 2010). Mais l'augmentation de la part relative de la catégorie alimentaire est aussi incontestablement liée à la croissance des couches moyennes salariées depuis les années 1960 et à la nature particulière de leurs habitudes de consommation: moyennes, elles aussi, mais résolument éclectiques et marquées par une forte volonté d'esthétisation du quotidien (Featherstone, 2007). Les lieux de consommation culinaire, en particulier les bars (Ernst \& Doucet, 2014; Luckins, 2009; Lugosi, Bell, \& Lugosi, 2010; Mathews \& Picton, 2014), les cafés et les restaurants, contribueraient ainsi à une importante restructuration du commerce des quartiers centraux dans les métropoles du tertiaire avancé.

Selon cette lecture, les nouveaux établissements alimentaires se distinguent des anciens par la nature de leurs marchandises et de leurs prestations. La cuisine y est davantage influencée par les gastronomies étrangères (Van Criekingen \& Fleury, 2006, p. 120) ; « la nourriture est cosmopolite, mais plus dans le sens de l'hybridité que de l'authenticité. Différence, fait-maison et gourmet en sont les principaux thèmes » (Bridge \& Dowling, 2001, p. 102, nous traduisons). Établi par la sociologue Sharon Zukin (1991, p. 212) dès le début des années 1990, le parallèle entre l'émergence d'une nouvelle cuisine et le retour aux quartiers anciens apparaît encore plus fondamental aujourd'hui, certains auteurs parlant même désormais de gentrification culinaire (Bell, 2004, p. 55) pour souligner le rôle central des classes moyennes urbaines dans la restructuration des hiérarchies de goûts et l'émergence de nouveaux types d'établissements de

\footnotetext{
2 Calculs réalisés par l'auteur à partir de l'annuaire montréalais des rues Lovell, 1971-72, 1977, 1982, 1987-88, 1992, 1997-98, 2006-07. Les données de 2011 ont été obtenues lors d’un recensement terrain.
} 
restauration. Cette transformation de l'offre commerciale apparaît donc étroitement liée à celle de la population, et plus précisément à l'augmentation du capital économique et culturel à l'intérieur de la zone naturelle de chalandise des commerces, un embourgeoisement que confirment d'ailleurs les statistiques socio-économiques disponibles pour les deux quartiers étudiés. ${ }^{3}$

Cet article mobilise un matériau empirique double: d'une part des données statistiques sur l'évolution du commerce des deux quartiers, compilées à partir d’anciens annuaires téléphoniques à chaque année de recensement pour la période 1971-2011 (Schlichtman \& Patch, 2008); d’autre part un corpus de 30 entretiens qualitatifs réalisés auprès de commerçants proposant un assortiment au moins en partie composé de produits ou de services alimentaires. ${ }^{4}$ Ces commerçants ont été recrutés dans leur magasin sur la base d’un échantillonnage non aléatoire réalisé à partir du registre des entreprises du Québec et diversifié quant à deux dimensions principales: l'ancienneté de l'établissement et le type de produits ou de services offerts. D’une durée moyenne d'un peu plus de 50 minutes, les entretiens ont principalement traité du parcours et des choix personnels et professionnels du propriétaire. L'approche était résolument inductive et encourageait chaque participant à baliser son univers social et professionnel, en lui demandant d'identifier ses fournisseurs, ses concurrents, ses clients et d'expliciter les critères discriminants mobilisés dans leur appréciation respective, de même que la nature et la fréquence de ses interactions avec chacun d'eux. Les extraits d'entretiens rapportés dans le texte sont anonymes. Les commerçants sont identifiés par un numéro ${ }^{5}$ et par l'ancienneté de leur établissement (les anciens sont en activité depuis plus de 10 ans).

\section{Résultats}

L’échantillon comprend 11 établissements de restauration: deux cafés, un bar et huit restaurants, dont certains faisaient aussi office de traiteurs. Du côté des biens, on compte quatre boulangeries-pâtisseries, six boucheries et neuf autres établissements spécialisés (fromagerie,

\footnotetext{
${ }^{3}$ Alors qu’il ne représentait que 63 pourcent de celui de la région métropolitaine au recensement de 1996, le revenu individuel moyen de la zone primaire de chalandise de la rue Ontario en frôlait les 75 pourcent en 2012, selon les données de l'Agence fédérale du Revenu. Celui de la zone primaire de la rue Notre-Dame a connu une évolution encore plus marquée, passant entre 1981 et 2012 de 62 pourcent à 90 pourcent du revenu individuel moyen de la RMR. L'évolution d'un indice de statut social calculé par Walks et Maaranen (2008, p. 87) témoigne par ailleurs d'une augmentation du capital culturel dans les deux quartiers. Dans Hochelaga-Maisonneuve, il passe de 0,36 au recensement de 1961 à 0,86 à celui de 2006. Dans le Sud-Ouest, l'écart est encore plus important car l'indice passe de 0,23 à 1,04 pendant la même période et dépasse donc désormais la moyenne montréalaise. Nous remercions les professeurs David Hulchanski et Alan Walks, de l’Université de Toronto, d'avoir partagé ces données avec nous dans le cadre du Projet de recherche sur les quartiers en transition.

${ }^{4}$ Sur un total de 50 entretiens auprès de commerçants de types divers.

${ }^{5}$ Les participants à l'enquête ont été numérotés de P01 à P50. Nous ne révélons pas le sexe des participants pour préserver leur anonymat.
} 
tisanerie, confiserie, vrac, et cetera). Les 30 participants ont des trajectoires personnelles et professionnelles variées (tableau 1): neuf sont des autodidactes, six sont des artisans ayant précédemment travaillé dans d’autres commerces comme chefs ou comme pâtissiers avant de se lancer à leur compte, cinq sont de jeunes entrepreneurs scolarisés dans des domaines divers et 10 sont arrivés au commerce au terme d'une réorientation de carrière, incluant cinq professionnels « défroqués » ayant quitté une carrière prospère d'avocat ou de fiscaliste par désir d'entreprendre ou pour vivre de leur passion pour la bière artisanale ou le café. 18 d'entre eux s’inscrivaient de façon marquée dans un créneau de qualité, c’est-à-dire que le propriétaire se distinguait par des efforts de promotion particulièrement élaborés mettant l'accent sur la qualité de la marchandise où qu'il militait, bien au-delà des murs de son établissement, à l'amélioration qualitative de l’offre commerciale de la rue ou du marché.

Tableau 1: Profil des participants

\begin{tabular}{|c|c|c|c|c|c|c|c|c|c|}
\hline \multicolumn{4}{|c|}{ ENTREPRISES } & \multicolumn{6}{|c|}{ PROPRIÉTAIRES } \\
\hline \multicolumn{2}{|c|}{ Ancienneté } & \multicolumn{2}{|l|}{ Structure } & \multicolumn{2}{|c|}{ Âge } & \multicolumn{2}{|c|}{ Formation } & \multicolumn{2}{|l|}{ Parcours } \\
\hline$>10$ ans & 9 & Solo & 8 & $<30$ & 4 & $\begin{array}{l}\text { Secondaire } \\
\text { régulier ou } \\
\text { moins }\end{array}$ & 5 & Traditionnel & 9 \\
\hline \multirow[t]{5}{*}{$<10$ ans } & 21 & Couple & 8 & $30-39$ & 10 & Technique & 13 & $\begin{array}{l}\text { Artisan à son } \\
\text { compte }\end{array}$ & 6 \\
\hline & & Famille & 8 & $40-49$ & 6 & Universitaire & 12 & $\begin{array}{l}\text { Jeune } \\
\text { entrepreneur }\end{array}$ & 5 \\
\hline & & Partenariat & 6 & $50-59$ & 6 & & & $\begin{array}{l}\text { Professionnel } \\
\text { défroqué }\end{array}$ & 5 \\
\hline & & & & $60-69$ & 3 & & & $\begin{array}{l}\text { Autre } \\
\text { réorientation }\end{array}$ & 5 \\
\hline & & & & $>70$ & 1 & & & & \\
\hline & 30 & & 30 & & 30 & & 30 & & 30 \\
\hline
\end{tabular}

Parmi ces derniers, environ une dizaine affichaient un niveau de prix significativement supérieur à la moyenne de leur quartier. Cette prépondérance du créneau qualité s’explique par la mainmise de la grande distribution sur les segments plus basiques de l'offre commerciale, particulièrement ancienne dans le cas du commerce alimentaire.

\section{La polarisation du commerce alimentaire}

La grande distribution alimentaire est implantée au Québec depuis le début du vingtième siècle (Théberge, 1985), mais les anciennes rues commerçantes conservent jusqu'aux années 1960 un certain monopole spatial sur une clientèle certes peu fortunée, mais relativement stable parce que 
captive et plus homogène (Polèse, 1978). Ce marché de proximité s’est considérablement érodé depuis, l'accroissement de la mobilité individuelle permettant aux citadins de s'approvisionner ailleurs que dans leur propre quartier. L’implantation de supermarchés au cœur même de la plupart de ces artères a en outre progressivement absorbé une partie importante de la demande alimentaire locale.

On constate aisément les effets de cette concentration du capital sur le tissu commercial des deux quartiers étudiés. Ainsi, alors qu’elles étaient autrefois nombreuses et bien réparties sur le territoire, les épiceries généralistes disparaissent rapidement à partir des années 1970, si bien qu'on n'en compte plus qu'un petit nombre sur chacune des deux artères 40 ans plus tard. Pour la seule décennie 1970, la chute est de 75 pourcent sur la rue Notre-Dame et de 50 pourcent sur la rue Ontario. Cette baisse est en partie compensée par l'augmentation du nombre de « dépanneurs »—quasi inexistants en 1971—et dont une grande partie semble d'ailleurs être le résultat d'une conversion des épiceries familiales dont ils occupent souvent les emplacements et conservent parfois le nom. Cette évolution est à notre connaissance assez peu documentée pour la période récente (Bélair, 1983, p. 9), mais un ancien épicier du quartier Saint-Henri laisse entendre qu’il s’agit d'une trajectoire répandue: « J'avais déjà eu un commerce. J’ai toujours été dans l'alimentation. On appelait ça une épicerie à l'époque, puis après ça on a viré ça en dépanneur. Comme tout le monde » (P49, Ancien).

Jusqu’en 1981, le nombre de commerces spécialisés décline également—quoiqu’à un rythme moindre que celui des épiceries généralistes. Dans le Sud-Ouest, le nombre des boucheries, boulangeries et autres petits établissements de bouche remonte cependant de près de 50 pourcent dans les décennies 1980 et 1990 avant de se stabiliser au début des années 2000. Sur l'ensemble de la période, il a augmenté de 40,9 pourcent. Dans Hochelaga-Maisonneuve, c’est surtout avec la réouverture du marché public municipal en 1995 que le nombre de petits commerces de bouche a recommencé à croître. Il surpassait en 2011 de 35,7 pourcent son niveau de 1971. Cette polarisation de plus en plus généralisée du commerce des biens témoigne de l’accaparement progressif du marché généraliste par la grande distribution, alors que les établissements plus petits se positionnent plutôt dans des niches distinctives: des horaires différents dans le cas des dépanneurs (Kirby, 1978; 1986), la qualité pour les petits commerces de bouche spécialisés et souvent plus haut de gamme. 
Tableau 2: Évolution des effectifs commerciaux des deux rues à l'étude, 1971-2011

\begin{tabular}{|c|c|c|c|c|c|c|c|c|c|}
\hline & & \multirow{2}{*}{1971} & \multirow{2}{*}{1981} & \multirow{2}{*}{1991} & \multirow{2}{*}{1996} & \multirow{2}{*}{2006} & \multirow{2}{*}{2011} & \multicolumn{2}{|c|}{$\Delta$ 1971-2011 } \\
\hline & & & & & & & & $\mathbf{n}$ & $\%$ \\
\hline \multirow{5}{*}{ 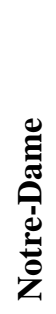 } & Alimentation & 39 & 28 & 34 & 40 & 43 & 43 & 4 & 2,6 \\
\hline & Générale & 17 & 6 & 5 & 5 & 6 & 6 & (11) & $(64,7)$ \\
\hline & Spécialisée & 22 & 19 & 23 & 30 & 30 & 31 & 9 & 40,9 \\
\hline & Dépanneurs & 0 & 3 & 6 & 5 & 7 & 6 & 6 & 600 \\
\hline & Restauration & 32 & 23 & 24 & 26 & 37 & 53 & 21 & 65,6 \\
\hline \multirow{5}{*}{$\begin{array}{l}0 \\
0 \\
0\end{array}$} & Alimentation & 25 & 23 & 31 & 34 & 34 & 35 & 10 & 40 \\
\hline & Générale & 8 & 6 & 4 & 5 & 5 & 3 & (5) & $(62,5)$ \\
\hline & Spécialisée & 14 & 12 & 17 & 17 & 14 & 19 & 5 & 35,7 \\
\hline & Dépanneurs & 3 & 5 & 10 & 12 & 15 & 13 & 10 & 333,3 \\
\hline & Restauration & 24 & 18 & 32 & 38 & 32 & 49 & 25 & 104,2 \\
\hline
\end{tabular}

Source: Calculs réalisés par l'auteur à partir de l'annuaire montréalais des rues Lovell, 1971-72, 1977, 1982, 1987 88, 1992, 1997-98, 2006-07. Les données de 2011 ont été obtenues lors d'un recensement terrain. Les données pour l'année 2001 ne sont pas disponibles.

\section{Des bas prix vers la qualité}

La grande distribution a accès à des économies d'échelle hors de la portée des petites entreprises. Rappelons simplement qu'en 2012 au Québec, la marge brute moyenne des magasins d'alimentation indépendants ne représentait que 80 pourcent de celle des magasins à succursales et leurs bénéfices nets d'exploitation, à peine plus de 13 pourcent. ${ }^{6}$ Ces économies contribuent à un abaissement des prix à un niveau inatteignable jusqu'alors, transformant durablement le marché de la vente au détail. En effet, si des chaînes comme Steinberg avaient toujours axé leur marketing sur les économies qu'elles faisaient réaliser aux consommateurs (Gibbon \& Hadekel, 1990), c’est bien dans les années 1980, après le rachat des magasins Dominion par Provigo, qu’a lieu à Montréal la première " guerre des prix » entre les grandes chaînes de supermarchés (Provost \& Chartrand, 1988, p. 202). C’est aussi dans les années 1980 et plus encore dans les années 1990 que se déploient dans la métropole les enseignes à rabais Super C et Maxi, aujourd'hui présentes dans plusieurs quartiers centraux et notamment sur les deux rues à l'étude. L'introduction récente de rayons alimentaires tant dans les chaines de pharmacies que dans les magasins de marchandises diverses tend également à accentuer la concurrence dans le segment

\footnotetext{
${ }^{6}$ Statistique Canada. Enquête annuelle sur le commerce de détail. Tableau 080-0023 : Estimations financières fondée sur le Système de classification des industries de l'Amérique du Nord (SCIAN) par genre de magasin, annuel. Calculs de l'auteur.
} 
de la nourriture bas de gamme, ces entreprises se spécialisant dans les produits à gros volume (Ministère de l'Agriculture, des Pêcheries et de l'Alimentation, 2015, p. 28). On assisterait dès lors au sein des quartiers centraux à la diffusion d'une nouvelle culture de consommation, différente par son envergure et ses moyens: multipliant les réclames, les « spéciaux » et les « circulaires » (Merrilees, McKenzie, \& Miller, 2007).

La croissance de la grande distribution alimentaire et son influence grandissante sur les réseaux d'approvisionnement rendent donc de plus en plus insoutenable la position des petits commerces indépendants, cela bien au-delà du seul commerce alimentaire. Dans les deux quartiers, ces établissements étaient souvent peu spécialisés et s’inscrivaient dans une logique de prix que plusieurs commerçants ont qualifié de « raisonnables ». Sans entrer dans la logique des aubaines et des produits d'appel vendus à perte - typique de la grande distribution et qu'ils auraient d'ailleurs bien du mal à imiter_plusieurs petits commerçants entendent ainsi rester concurrentiels « en moyenne », une stratégie particulièrement répandue chez les bouchers établis de longue date:

Je te dirais que dans nos prix, c'est très ressemblant à ce qu'on doit avoir dans les chaînes, à part les items qui sont en spécial, qu'eux autres vendent en bas du prix coûtant pour attirer une clientèle. Mais nos prix, en règle générale, sont les mêmes que dans les chaînes, même que parfois on vend moins cher. ${ }^{7}$ Mais ça, les gens ne le savent pas... Quand le [supermarché à rabais] Super $C$ a ouvert il y a quelques années, on s'est dit qu'ils allaient nous tuer. Et finalement ça n’a même pas paru. C’est pas la même clientèle. Ici on a une spécialité de viandes fraîches. Super $C$, ce n'est pas du tous les mêmes gens... C'est surtout des BS et nous on n'en a pas vraiment. Ils vont chercher les ventes... (P12, Ancien).

On constate l'ambiguïté de la position de ce commerçant qui juge ses prix concurrentiels avec ceux de la grande distribution, tout en indiquant un peu plus loin dans l'entretien ne pas desservir la clientèle la plus pauvre, celle des assistés sociaux. Dans son commerce établi de longue date, ce boucher indique par ailleurs ne pas avoir radicalement transformé son offre au fil des ans. Ses propos suggèrent plutôt que ce serait le marché de la viande qui se serait polarisé entre des grandes surfaces et des supermarchés, d'une part qui vendent toujours moins cher une viande préemballée non transformée sur place, et un ensemble de petits établissements plus haut-degamme, d'autre part.

Au marché Atwater, dans le Sud-Ouest, certains bouchers sont ainsi nettement plus actifs dans cette dernière logique et multiplient les marqueurs de distinction: viande biologique, réseaux de petits producteurs, coupes françaises, personnel européen. Mais tous s’inscrivent sans forcément l'avoir cherché dans un créneau nostalgique de boucherie « à l’ancienne » par leur

\footnotetext{
${ }^{7}$ Cette affirmation semble toutefois contredite par des enquêtes d'associations de consommateurs réalisées au marché Atwater, où est installé ce commerçant (Dô, 2005, Juin).
} 
seule présence dans cet imposant bâtiment patrimonial et par leur allure «traditionnelle » : comptoirs à l'ancienne, sarraus blancs, viande empaquetée sur place dans un papier rose plutôt qu'en emballage sous vide, enseignes vieillottes. L’importante baisse des prix menée par la grande distribution aurait donc de facto exclu de la catégorie des bas prix tous ceux qui cherchent à rester concurrentiels « en moyenne », creusant la gamme vers le bas à un niveau où les indépendants ne peuvent suivre en dépit des efforts de certains pour demeurer accessibles au plus grand nombre. De plus en plus, la logique des bas prix « raisonnables » des petits commerçants apparaît résolument incompatible avec celle des « aubaines » et du « discount » pratiquée par la grande distribution, dont les « spéciaux » et les produits d'appel s’apparentent souvent à une forme de dumping (Péron, 2004).

On observe également une évolution des habitudes de consommation qui tend à réduire le volume des ventes, une diminution que les bouchers sont encore une fois parmi les premiers à constater. En effet, la consommation de viande rouge a diminué dans les dernières décennies en raison de multiples facteurs: expansion du marché des plats préparés, diminution de la taille des familles, préoccupation croissante pour l'alimentation et la santé, popularité du végétarisme et du végétalisme, généralisation des peurs alimentaires dans le sillage des épidémies de grippe aviaire ou de vache folle (Fischler, 2001; Freidberg, 2004), crainte de ce que le sociologue Claude Fischler (1990, p. 209) a qualifié d'objets comestibles non identifiés, et cetera. Plusieurs bouchers observent ainsi que « les gens ne font plus de commandes comme dans le temps » (P17, Nouveau), une réalité sans doute liée à l'augmentation du nombre de ménages d'une seule personne et qui affecterait grandement la rentabilité de leur commerce: « Avec des petites portions c’est plus difficile d'avoir un total élevé qu’avec des grosses portions. Par contre, c'est normal, les familles sont plus petites. Donc il faut travailler plus pour vendre ce qu'on vend, qu'autrefois quand j'ai commencé. Avant, c’était toutes des grosses commandes » (P19, Ancien).

Qu’on soit petit ou grand, maintenir un niveau de prix bas suppose en effet, pour générer un bénéfice raisonnable, un certain volume de ventes. La diminution de la consommation rend ce positionnement de plus en plus intenable pour les petites entreprises, plusieurs étant dès lors contraintes d'augmenter leurs marges pour compenser la baisse de volume: « Ça prend du volume... C'est bien beau vendre des petites affaires, là... C'est ça le problème aujourd'hui, c'est que tu as moins de volume. Mais c'est la vie et il faut s'adapter à ça... Tu montes tes prix. J’ai toujours haï ça monter les prix... Mais des fois, t’as pas le choix » (P12, Ancien).

\section{Réseaux de fournisseurs alternatifs et constitution d'un assortiment original et distinctif}

Pour être considéré légitime et donc accepté par le consommateur, un niveau de prix plus élevé doit offrir une contrepartie, entendue ici de façon large comme une qualité socialement valorisée. C’est sans doute le grand intérêt de la petite production alternative pour ces commerçants. De tels réseaux leur permettent en effet de se distinguer de la grande entreprise, de se poser en alternative attrayante à des modes de distribution de plus en plus critiqués par une frange de la 
population (Quéniart, Jacques, \& Jauzion-Graverolle, 2007). Le succès des petits commerces reposerait donc de plus en plus sur la singularisation et le niche marketing, des stratégies qui s’inscrivent dans un déclin plus général de la production de masse dans les économies du tertiaire avancé, au profit d'un capitalisme renouvelé par une marchandisation de la différence (Boltanski et Chiapello, 1999, p. 592) et caractérisé par une très grande variété de produits et surtout de services de consommation. Comme l'a résumé le propriétaire d'un café indépendant suite à l'ouverture d'une grande chaîne à proximité de son établissement, « être à côté d'un Tim Hortons, c'est moins mauvais que j'aurais pensé parce qu'il y a quand même plein de monde qui ne veulent pas s'associer à cette culture-là » (P03, Nouveau).

À travers leur assortiment atypique, local ou traditionnel, ces commerçants se rattachent ainsi à des univers valorisés par la nouvelle clientèle mieux nantie: « Les gens qui vont entrer ici ne veulent pas manger un hamburger, ils veulent manger un bon « grilled cheese » avec du jambon bio, du fromage qui vient de l'île-aux-Grues avec du thym bio et ils sont prêts à payer le prix... c’est ces gens-là qu’on vise » (P25, Nouveau).

On vend de la qualité, mais avec la qualité vient un certain prix. Je pense que les consommateurs et les commerçants, on a évolué ensemble. Il y en a pour qui ça a été un peu plus long, mais les commerçants se sont ajustés au niveau de la qualité de ce qu'ils proposaient aux clients. La clientèle aussi s’est « améliorée », les Québécois ont voyagé... ils revenaient d’Europe et disaient: « on a goûté à telle ou telle affaire, peux-tu nous en avoir? ». Fait que c'était à nous, les commerçants, de faire des recherches et puis ça s'est fait progressivement. Je prends un exemple: du baloney, on n'en vend plus parce que les gens ne nous en demandent plus (P23, Ancien).

La disparition du baloney, le « saucisson de Bologne », pilier traditionnel de l'alimentation populaire québécoise, marque ici symboliquement cette évolution de la clientèle, le passage d'une alimentation bourrative à une culture gastronomique plus sophistiquée que reflète aujourd'hui l'assortiment de cet établissement, composé essentiellement de produits du terroir québécois et d’importations européennes.

On constate le rôle central que jouent désormais les petits commerçants dans la rencontre d'une offre et d'une demande de plus en plus pointues. En effet, le régime de concurrence monopolistique dans lequel cherchent à s'inscrire plusieurs de ces commerçants se caractérise par un renouvellement accéléré et continu des pratiques de vente. Le marché des produits de niche est plus volatile que celui de la consommation courante, la demande étant plus que jamais façonnée par les modes et le marketing. Pour les produits très spécialisés, la valeur repose en effet sur des critères très subjectifs de qualité. Elle est donc largement sociale et dépend de la capacité du commerçant à convaincre le consommateur, à jouer le rôle d’impresario de « ses » producteurs, de banquier symbolique de sa marchandise (Bourdieu, 1977, p. 6). En France, le sociologue Lucien Karpik a ainsi montré comment, « à côté d’une économie classique composée 
de biens standardisés dont les propriétés sont connues du consommateur, se développe la sphère des biens et des services qui se caractérisent par l'incomplétude et qui ne peuvent être réellement définis qu'après l'achat » (Karpik, 1989, p. 206). C’est ce qu'il appelle le marché des singularités, qui se caractérise par des « relations marquées par l’incertitude sur la qualité [de] produits singuliers » entre des vendeurs et des acheteurs à la recherche de la « bonne singularité » (Karpik, 2007, p. 38). Ce qui distingue ce marché des autres est donc ce qui fait son intérêt pour les offrants, à savoir une « asymétrie de l’information favorable au producteur » (Karpik 2007, p. 42), qui peut alors tirer profit de « situations dans lesquelles le client, à la différence du vendeur, a du mal à distinguer la mauvaise qualité de la bonne, alors que pour lui cette distinction est importante » (Karpik, 1989, p. 202).

Dans ce contexte, nombreux sont les commerçants alimentaires qui mettent de l'avant leur relation particulière à leurs fournisseurs. Ainsi dans une boucherie du marché Atwater, on peut voir défiler, sur un écran de télévision placé en évidence à l'entrée de l’étal, les fermes et les producteurs dont les viandes sont distribuées sur place. Au même numéro, la propriétaire affiche fièrement une plaque rappelant son statut d'agricultrice de l'année. En face, une boucherie se spécialise dans les viandes biologiques et l'indique clairement sur son enseigne. Ces discours sans doute sincères peuvent toutefois s'avérer assez superficiels. Un boulanger va ainsi importer du beurre de Nouvelle-Zélande et plusieurs commerçants distribuent une grande variété de produits importés, même si on essaie à travers un ensemble de subterfuges de les rendre aussi vertueux que possible. Lorsque les produits ne peuvent être locaux, pour des raisons souvent climatiques, certains commerçants usent de procédés discursifs pour légitimer leur provenance, idéalement en la valorisant dans un curieux mélange de localisme et de cosmopolitisme: « On choisit que des produits du terroir, et ce qui n'est pas du terroir, mettons l'huile d'olive... il n'y en a pas au Québec, mais il y a la belle excuse, c’est une Québécoise mariée avec un Grec qui y a des oliviers en Grèce et ils partent six mois par année chercher l’huile. C’est la plus québécoise des huiles d'olive » (P02, Nouveau).

Cette mise en récit témoigne de la grande flexibilité avec laquelle le principe de traçabilité est mobilisé pour pallier certains accrocs inévitables à l'approvisionnement local. Tous ces éléments—proximité, taille restreinte, caractère artisanal, exotisme—sont d'ailleurs souvent associés jusqu'à se confondre dans les discours, une vague référence à l'Europe venant souvent cautionner et incarner cet idéal de proximité: «C’est une boutique 100 pourcent locale, québécoise. Je mise vraiment sur les régions ressource... Toutes les régions qui ont un taux de chômage plus élevé, on essaie de les pousser vraiment. C’est grâce à eux qu'on mange bien » (P08, Nouveau).

Que ce commerçant fasse référence aux « régions ressources » ayant traditionnellement occupé une place très marginale dans l'agriculture québécoise en raison de leur climat particulièrement rigoureux témoigne sans doute d'une vision en partie romancée de la ruralité et de la production alimentaire. Mais même romancée, celle-ci s’appuie sur des principes éthiques et esthétiques forts qui poussent cet entrepreneur à développer des liens avec ces territoires en déclin. On constate ici que ce positionnement n'est pas qu'un slogan publicitaire, mais qu'il peut 
aussi prendre sa source dans des valeurs personnelles des commerçants, dont plusieurs abordent l'univers de la boutique avec un ensemble de valeurs non strictement entrepreneuriales, témoignant même d'une forte idéologie progressiste et environnementaliste. Tel est notamment le cas de cette boulangère: « On remonte jusqu’au producteur parce qu’on croit beaucoup à la naturalité et on croit qu'il ne faut pas faire les choses comme les gros commerciaux le font, même si on est gros. On connaît les agriculteurs qui produisent notre blé. On sait où ils sont, on a vu leur champs » (P38, Ancien).

\section{Contraintes et difficultés d'un approvisionnement alternatif}

Au-delà de la valeur marchande et des principes moraux, il y a également des avantages nettement plus prosaïques pour un commerçant à s'associer à des petits producteurs locaux. Avoir un réseau stable de fournisseurs situés à proximité permet de négocier plus facilement les quantités, les frais et les dates de livraison ou même d'aller chercher la marchandise soi-même. Mais ces assortiments uniques, aussi originaux et différents que possible, compliquent aussi considérablement le travail des commerçants qui doivent pour les composer faire preuve d'une grande inventivité et investir considérablement de temps et de ressources dans la constitution de réseau de petits fournisseurs souvent nettement moins prévisibles. En effet, la constitution d'un tel réseau implique souvent la participation à des associations ou à des réseaux institutionnels comme la Guilde des herboristes ou l'Union paysanne, l’abonnement à des revues spécialisées ou à des groupes sur les réseaux sociaux en ligne: « Il y a un congrès annuel ou je peux en rencontrer. Parfois aussi, à partir d'un producteur j’ai réussi à en connaître d'autres parce qu'il dit: « J'en en ai plus mais peut-être qu'elle, elle en aurait ». Donc à partir de là on développe une relation. Ça fonctionne vraiment par relations là-dedans, parce qu’ils sont rarement affichés sur Internet. Comme ils ne sont pas gros, souvent ils ne vendent que sur leurs terres et ce sont des gens qui les connaissent qui vont acheter leurs produits » (P47, Nouveau). En témoigne également ce restaurateur:

Ça reste très artisanal comme milieu. Une fois que t'es rentré, tu te ramasses avec des fournisseurs qui sont toujours en stock limité. Alors ils se mettent à livrer à du monde qu'ils aiment bien ou à du monde qu'ils savent qu'ils vont faire un bon travail. Et en tant qu'acheteur, il y a une situation de rareté ou moi je suis un peu poigné. C’est moi qui les appelle pour qu'ils me vendent des trucs. Mais en même temps ça permet de développer des relations particulières qui vont faire que tels produits on est les seuls à les avoir et il y a du monde qui vont venir chez nous pour ça (P20, Nouveau).

On constate bien ici tout le paradoxe de ce positionnement, en ce que la rareté- et donc la valeur-des avantages qu'il confère est directement liée à la difficulté de son établissement. 
La spécialisation d'une boutique ou d'un magasin dépend largement de l'originalité de son assortiment et, partant, de son réseau de fournisseurs qui permet au commerçant de s'approprier une partie du capital symbolique des producteurs. Plus ceux-ci sont petits, lointains, artisanaux, traditionnels — tout ce qui permet de les distinguer de la production industrielle de masse-est valorisé, perçu comme intrinsèquement positif. Il s'agit également d'une prise de distance vis-àvis des rendements et de l'efficacité de la production industrielle.

C’est évidemment dans les commerces d'aliments périssables que cet approvisionnement s’avère le plus délicat. Plus le producteur est petit, plus ses méthodes sont naturelles, plus la production est susceptible de fluctuer dans le temps. Les bouchers doivent donc manœuvrer de manière à préserver des liens de confiance et ne pas fragiliser leurs producteurs, tout en assurant une offre stable à leur clientèle: « Je suis fidèle. Tu sais, tu peux pas acheter de plein de monde. C’est pas de même que ça marche. Il faut qu'il y ait une loyauté et une parole. Mais t'en prends toujours deux parce que s'il y en a un qui a une 'bad luck,' toi tes clients qui ont réservé des dindes, ils n'en auront plus pour Noël ou pour l'Action de grâces. Donc j'en ai un qui me livre tel jour et l'autre tel autre jour. On se sécurise » (P17, Nouveau).

La saisonnalité des produits constitue aussi un défi, car la clientèle des commerçants urbains n'est souvent pas aussi fidèle et convaincue que peut l'être celle d'autres réseaux alternatifs, comme l'agriculture soutenue par la communauté (Quéniart et al., 2007, p. 188). Il devient dès lors difficile pour le commerçant de concilier la préservation des aliments et les normes sanitaires, d'une part, et les exigences esthétiques de clients comme les présentoirs ou les emballages à l'ancienne, d'autre part. La gestion des stocks est donc plus complexe qu'elle ne l'était quand les habitudes de consommation étaient à la fois plus régulières-les ménagères faisaient un marché hebdomadaire voir même mensuel de viande-et plus standardisées. En témoigne ce boucher: «C’est un fait, les gens veulent savoir ce qu’ils mangent. Mais ça ne veut pas dire qu'ils achètent plus. Ça prend du volume... C’est bien beau vendre des petites affaires, là... Il faut que tu en vendes en maudit des magrets de canard pour te faire une paye, pour payer ton stock, pour payer ton loyer, pour payer tes fournisseurs » (P12, Ancien).

\section{Conclusion}

Ce qui fait l'originalité de la viennoiserie, du repas gastronomique au restaurant ou de la tisane biologique relève tout autant du travail du commerçant que de celui du producteur de la matière première. C'est à travers l'ensemble de la chaîne de distribution que la singularité des biens et des services_c'est-à-dire ce qui constitue leur caractère alternatif à la production de masse-est produite et surtout valorisée de manière à s’insérer dans la sphère de la consommation. Car si la qualité perçue dépend bien de caractéristiques concrètes, elle procède aussi largement d'une mise en récit et d'efforts variés de promotion visant à communiquer ces caractéristiques et à les rendre assimilables et crédibles aux yeux des consommateurs. On sait en effet que ceux-ci misent « sur la confiance envers le commerçant ou le producteur qui devient même, dans certains cas, 
équivalente et parfois supérieure à la certification [...], certains n’hésitant pas à acheter des produits qui ne sont pas certifiés par un organisme, se fiant à la parole du commerçant qu'ils connaissent et avec qui ils peuvent échanger » (Quéniart et al., 2007, p. 187). C’est donc la proximité sociale et géographique des petits commerçants avec les classes moyennes urbaines qui leur permet d'agir comme intermédiaires, assurant en aval une médiation entre les goûts des consommateurs et la nouvelle production locale dont ils se font ainsi les porte-voix. Ils apparaissent de plus en plus influents en dépit de leurs ressources limitées, comme l'a d'ailleurs montré Manon Boulianne dans le cas des filières fromagères qui dépendraient tout autant des circuits courts que des « détaillants spécialisés qui connaissent personnellement les fromagers et fromagères, ainsi que leurs produits et les procédés de fabrication auxquels ils recourent, et qui peuvent en informer la clientèle » (Boulianne, 2010, para. 12).

Il apparaît difficile, au terme de ce survol, de définir a priori et donc d'essentialiser le caractère alternatif tant de la petite production que de la distribution alimentaire. Il est en effet dans la nature de certaines entreprises de croître et d'éventuellement compter parmi les figures dominantes de leurs industries respectives. L'absorption de petits producteurs fromagers par la coopérative Agropur (Beauchamp, 1988, p. 243), le rachat de microbrasseries locales comme Unibroue par les géants Sleeman et Sapporo ou encore l’intégration récente des supermarchés ethniques Adonis et des boulangeries-pâtisseries Première Moisson au sein de Métro témoignent de la capacité de la grande distribution à absorber progressivement les petites entités innovantes. Il semble ainsi plus fécond de définir la distribution alimentaire alternative non pas comme le prolongement ou le canal d'écoulement d'une production alimentaire alternative, mais comme étant elle-même une alternative à la grande distribution, de la même manière que la production alternative est une alternative à l'industrie agroalimentaire aujourd'hui dominante. Producteurs et distributeurs alternatifs constituent ainsi deux maillons d'une chaîne qui ne peut donc être qualifiée d'alternative que tant qu'elle s'attache à se démarquer de l'univers de la consommation de masse.

Aborder les différents acteurs du processus de distribution comme composante d'une chaîne, d'un réseau ou d'une filière suppose la prise en compte de chacun des éléments qui composent ces collectifs, de même que la nature et la force des liens qui les unissent. C'est ce que nous avons tenté de faire dans cet article, en examinant les réseaux alimentaires alternatifs du point de vue des petits commerçants urbains. Ce regard particulier est lui-aussi partiel et bénéficierait d'un examen approfondi des interactions survenant entre les détaillants et les consommateurs, notamment pour comprendre comment la confiance est produite sur un temps long, non seulement à travers des dispositifs conscients mais aussi par un ensemble de liens moins strictement économiques de voisinage. 


\section{Bibliographie}

Association des marchés publics du Québec. (2014). Caractérisation des marchés publics membres de l'AMPQ. Québec: Association des marchés publics du Québec et Ministère de l'agriculture, des pêcheries et de l'alimentation.

Baudrillard, J. (1972). Pour une critique de l'économie politique du signe. Paris: Gallimard.

Beauchamp, C. (1988). Agropur: cinquante ans de rêves et de réalisations depuis la Société coopérative agricole du canton de granby, 1938-1988. Montréal: Boréal.

Bélair, G. (1983). Chronologie des épiciers en gros et des détaillants au Québec. Montréal: Association des épiciers en gros de la province de Québec inc.

Bell, D. (2004). Taste and Space: Eating out in the City Today. Dans D. Sloan (Ed.), Culinary taste: consumer behaviour in the international restaurant sector. (pp. 43-58). Boston: Elsevier.

Bérard, L., et Marchenay, P. (2004). Les produits de terroir: entre cultures et règlements. Paris: Éditions du CNRS.

Bergeron, Y. (1990). Les anciennes halles et places de marché au Québec : étude d'ethnologie appliquée (Thèse de doctorat non publiée). Université Laval, Québec.

Blay-Palmer, A., et Donald, B. (2006). A Tale of Three Tomatoes: The New Food Economy in Toronto, Canada. Economic Geography, 82(4), 383-399.

Boltanski, L. et Chiapello, E. (1999). Le Nouvel esprit du capitalisme, Paris: Gallimard.

Boulianne, M. (2010). Valorisation des terroirs et économie morale au 21e siècle : le cas des fromages fins du Québec. Cuizine: The Journal of Canadian Food Cultures, 2(2). https://doi.org/10.7202/044350ar.

Boulianne, M. (2013). Artisans du pays et imaginaires fromagers : La qualification des fromages fins du Québec comme produits de terroir. Anthropologie et Sociétés, 37(2), 213-231.

Bourdieu, P. (1977). La production de la croyance: contribution à une économie des biens symboliques. Actes de La Recherche En Sciences Sociales, 13(1), 3-43. 
Bridge, G., et Dowling, R. (2001). Microgeographies of Retailing and Gentrification. Australian Geographer, 32(1), 93-107.

Brouillette, S. (1991). Les marchés publics à Montréal, 1840-1860 (Mémoire de maîtrise non publié). Université du Québec à Trois-Rivières.

CAAAQ. (2008). Agriculture et agroalimentaire : assurer et bâtir l'avenir. Québec: Commission sur l'avenir de l'agriculture et de l'agroalimentaire québécois.

Callon, M., Méadel, C., et Rabeharisoa, V. (2000). L’économie des qualités. Politix, 13(52), 211-239.

Condon, G. (2016, Mars). State of the Grocery Nation: Annual Market Survey. Canadian Grocer, pp. 26-28.

De Koninck, R. (1993). La vigne et le vin au Québec: bon goût et ténacité vigneronne. Cahiers de Géographie Du Québec, 37(100), 79-111.

Dô, S. (2005, Juin). Paniers à surprise. Protégez-Vous, pp. 28-29.

Ernst, O., et Doucet, B. (2014). A Window on the (Changing) Neighbourhood: The Role of Pubs in the Contested Spaces of Gentrification. Tijdschrift Voor Economische En Sociale Geografie, 105(2), 189-205.

Featherstone, M. (2007). Consumer culture and postmodernism (2e éd.). Londres: Sage.

Fischler, C. (1990). L’omnivore. Le goût, la cuisine et le corps. Paris: Odile Jacob.

Fischler, C. (2001). La peur est dans l’assiette. Revue Française du Marketing, (183/184), 7-10.

Fligstein, N. (1996). Markets as politics: A political-cultural approach to market institutions. American Sociological Review, 61(4), 656-673.

Freidberg, S. (2004). French Beans and Food Scares: Culture and Commerce in an Anxious Age. Oxford: Oxford University Press.

Gibbon, A., et Hadekel, P. (1990). Steinberg: the breakup of a family empire. Toronto: Macmillan. 
Gonzalez, S., et Waley, P. (2013). Traditional retail markets: The new gentrification frontier? Antipode, 45(4), 965-983.

Groupe Marché. (2007). La dynamique de la distribution alimentaire, d'hier à aujourd'hui. Québec: MAPAQ, Direction des études économiques.

Guthman, J. (2004). Agrarian dreams: the paradox of organic farming in California. Berkeley: University of California Press.

Hess, D. J. (2009). Localist movements in a global economy: sustainability, justice, and urban development in the United States. Cambridge, MA: MIT Press.

Johnston, J., et Baumann, S. (2010). Foodies: democracy and distinction in the gourmet foodscape. New York: Routledge.

Karpik, L. (1989). L’économie de la qualité. Revue Française de Sociologie, 30(2), 187-210.

Karpik, L. (2007). L'économie des singularités. Paris: Gallimard.

Kirby, D. (1978). What happened to the local grocer? Retail and Distribution Management, 6(5), 52-55.

Kirby, D. (1986). Convenience stores: the polarisation of British retailing. Retail and Distribution Management, 14(2), 7-12.

La Pradelle, M. de. (1996). Les vendredis de Carpentras: faire son marché en Provence ou ailleurs. Paris: Fayard.

Lemasson, J.-P. (2006). Montréal, capitale gastronomique? Téoros. Revue de recherche en tourisme, 25(1), 24-31.

Luckins, T. (2009). Gentrification and Cosmopolitan Leisure in Inner-Urban Melbourne, Australia, 1960s-1970s. Urban Policy and Research, 27(3), 265-275.

Lugosi, P., Bell, D., et Lugosi, K. (2010). Hospitality, Culture and Regeneration: Urban Decay, Entrepreneurship and the “Ruin” Bars of Budapest. Urban Studies, 47(14), 3079-3101.

Mathews, V., et Picton, R. M. (2014). Intoxifying gentrification: brew pubs and the geography of post-industrial heritage. Urban Geography, 35(3), 337-356. 
Merrilees, B., McKenzie, B., et Miller, D. (2007). Culture and marketing strategy in discount retailing. Journal of Business Research, 60(3), 215-221.

Ministère de l'Agriculture, des Pêcheries et de l'Alimentation. (2015). Bottin statistique de l'alimentation. Québec: MAPAQ, Direction générale des politiques agroalimentaires.

Paxson, H. (2013). The life of cheese: crafting food and value in America. Berkeley: University of California Press.

Péron, R. (2004). Les boîtes: les grandes surfaces dans la ville. Nantes: L’Atalante.

Polèse, M. (1978). Le commerce et la distribution au Québec : le tissu urbain québécois. Montréal: INRS Urbanisation.

Provost, R., et Chartrand, M. (1988). Provigo. Le plus grand succès de l'entrepreneurship québécois. Montréal: Éditions de l’Homme.

Quéniart, A., Jacques, J., et Jauzion-Graverolle, C. (2007). Consommer autrement : Une forme d'engagement politique chez les jeunes. Nouvelles pratiques sociales, 20(1), 181-195.

Robitaille, J. (2012). La consommation de viande: évolution et perspectives de croissance. Québec: Ministère de l'agriculture, des pêcheries et de l'alimentation, Direction des études et des perspectives économiques.

Savage, M., Bagnall, G., et Longhurst, B. (2005). Globalization and belonging. Thousand Oaks: Sage.

Schlichtman, J., et Patch, J. (2008). Contextualizing impressions of neighborhood change: Linking business directories to ethnography. City \& Community, 7(3), 273-293.

Théberge, G. (1985). L'évolution de la distribution alimentaire au Québec de 1930 à 1984. Montréal: École des hautes études commerciales.

Trubek, A. B. (2009). The taste of place: a cultural journey into terroir. Berkeley: University of California Press.

Tunbridge, J. E. (2001). Ottawa’s Byward Market: A festive bone of contention? The Canadian Geographer/Le Géographe Canadien, 45(3), 356-370. 
Van Criekingen, M., et Fleury, A. (2006). La ville branchée : gentrification et dynamiques commerciales à Bruxelles et à Paris. Belgeo. Revue belge de géographie, (1-2), 113-134.

Walks, R. A., et Maaranen, R. (2008). The timing, patterning, and forms of gentrification and neighbourhood upgrading in Montreal, Toronto, and Vancouver, 1961 to 2001. Toronto: Cities Centre, University of Toronto.

Zukin, S. (1991). Landscapes of power from Detroit to Disney World. Berkeley: University of California Press. 